\title{
ESTRATEGIAS COGNITIVAS Y METACOGNITIVAS EN LA COMPRENSIÓN DE UN TEXTO DE CIENCIAS
}

\author{
MATURANO, CARLA INÉS, SOLIVERES, MARÍA AMALIA y MACÍAS, ASCENSIÓN \\ Instituto de Investigaciones en Educación en las Ciencias Experimentales \\ Facultad de Filosofía, Humanidades y Artes. Universidad Nacional de San Juan \\ Av. J.I. de La Roza, 230 oeste. 5400 San Juan. Argentina \\ cmatur@ffha.unsj.edu.ar \\ msoliver@ffha.unsj.edu.ar \\ amacias@ffha.unsj.edu.ar
}

\begin{abstract}
Resumen. En este trabajo damos a conocer los resultados de la investigación que hemos realizado sobre algunas estrategias cognitivas y metacognitivas que usan los alumnos universitarios de diferentes carreras (Licenciatura en Geografía, Profesorado en Física, Profesorado en Química, Ingeniería Química y Bioingeniería) en la comprensión de un texto de carácter expositivo, extraído de un libro de física.

Palabras clave. Estrategias, cognición, metacognición, textos científicos.
\end{abstract}

Summary. In this paper we offer the results of a research made about some cognitive and metacognitive strategies used by university students from different degrees (Geography, Physics, Chemistry, Chemistry Engineering and Bioengineering) when reading an expository text taken from a Physics textbook.

Keywords. Strategies, cognition, metacognition, science texts.

\section{INTRODUCCIÓN}

Los textos expositivos son característicos de la mayoría de los textos de ciencias. Los alumnos de nivel universitario usan dichos textos para aprender y los docentes pretendemos que éstos sepan leerlos aplicando estrategias cognitivas y metacognitivas que les permitan concretar aprendizajes a través de ellos. Dichas estrategias involucran procesos relacionados con la cognición y la metacognición, acotados en este trabajo al aprendizaje a partir de textos científicos.

Considerando la opinión de investigadores en este campo, vemos que algunos autores como Brown, Armbruster y Baker (1986), Baker (1994), Campanario (1994), Martí (1995), Otero (1990, 1995, 1997) y Palincsar y Brown (1997), entre otros, han estudiado las dificultades de los estudiantes en la comprensión y retención de la información importante que provee un texto.
Flavell (1996) afirma que la función principal de una estrategia cognitiva es ayudar a alcanzar la meta de cualquier empresa cognitiva y una estrategia metacognitiva tiene como función informar sobre la empresa o el propio progreso. Las primeras ayudan a hacer un progreso cognitivo y las segundas a controlarlo. Nos interesa saber cómo se dan algunos procesos cognitivos y metacognitivos al trabajar con textos científicos y cuáles son los mecanismos que ponen en juego los estudiantes en la comprensión de un texto corto de carácter expositivo. Entendemos por comprender, la capacidad de hacer con un tópico una variedad de cosas que estimulan el pensamiento, como son explicar, demostrar, ejemplificar, generalizar, volver a presentar el tópico de otra manera, entre otras (Blythe y Perkins, 1999). En este trabajo analizamos las actividades que realizan los alumnos con el fin de poder sugerir acciones tendientes a mejorar los 
mecanismos de comprensión y damos a conocer el estudio que hemos llevado a cabo sobre estos aspectos.

\section{ESTRATEGIAS COGNITIVAS}

La cognición implica conocimiento, acción y efecto de conocer. El conocer es definido, en su acepción de sentido común, como averiguar por el ejercicio de las facultades intelectuales la naturaleza, cualidades y relaciones de las cosas (Diccionario de la Real Academia Española, 1992). Podemos precisar más esta definición con lo expresado por Gellatly (1997), quien afirma que la cognición se refiere a las actividades de conocer, es decir, recoger, organizar y utilizar el conocimiento.

Las operaciones cognitivas involucradas en la comprensión lectora incluyen reconocimiento de letras y su integración en sílabas; codificación de palabras; codificación sintáctica; codificación de proposiciones e integración temática para construir un modelo coherente e integrado del texto global (De Vega, 1993). Para ello, el sujeto debe buscar relaciones entre partes de una materia (relacionar), distinguir puntos secundarios y principales (seleccionar), pensar ejemplos (concretizar) y buscar aplicaciones (aplicar). Todas estas actividades hacen al procesamiento cognitivo (Vermut, 1996).

Luque y otros (1999) sostienen que las diversas representaciones que se construyen de un texto toman parte en las múltiples operaciones cognitivas que la comprensión requiere. El resultado de las operaciones cognitivas es la construcción de una estructura que integra elementos procedentes del texto con otros recuperados de la memoria. Así, el lector ajusta los nuevos conocimientos con los conocimientos previos.

Para Palincsar y Brown (1997), un buen lector es una persona que posee un repertorio de estrategias cognitivas que es capaz de usar de manera flexible durante la lectura. El lector experto tiene al menos un conocimiento procedimental sobre estrategias cognitivas más sofisticadas, como sería, por ejemplo, la de emplear más tiempo en estudiar materiales más importantes y menos conocidos que en estudiar los menos importantes y más conocidos (Flavell, 1996).

\section{ESTRATEGIAS METACOGNITIVAS}

La metacognición se refiere a «cualquier conocimiento o actividad cognitiva que tiene como objeto, o regula, cualquier aspecto de cualquier empresa cognitiva» (Flavell, 1996, p. 157). Para Palincsar y Brown (1997), el conocimiento metacognitivo permite al lector seleccionar, emplear, controlar y evaluar el uso de estrategias lectoras. Éstas implican, entre otras cosas, el monitoreo activo y la regulación posterior de las actividades de procesamiento de la información.
Baker (1994) sostiene que en el proceso de lectura existen dos actividades autorregulatorias importantes: la primera implica darse cuenta de si hemos entendido (evaluación) y la segunda consiste en dar los pasos adecuados para resolver los problemas de comprensión detectados (regulación). Esta última actividad sólo se pone en marcha cuando la comprensión resulta insatisfactoria (Otero, 1992). Según Baker (1994), para darse cuenta de si un texto se ha comprendido hay que utilizar una serie de criterios que corresponden a la fase de evaluación. Los mismos consisten en verificar:

- Criterio léxico: la comprensión del significado de cada palabra.

- Criterio de coherencia externa: que las ideas del texto y los conocimientos previos del lector sean compatibles.

- Criterio de cohesión proposicional: la cohesión local entre las ideas del texto.

- Criterio de cohesión estructural: la compatibilidad temática de las ideas del texto.

- Criterio de coherencia interna: la consistencia lógica de las ideas del texto.

- Criterio de suficiencia informativa: que el texto contenga la información necesaria para cumplir determinado objetivo.

Como ejemplos de estrategias metacognitivas podemos citar: ser conscientes de lo que uno sabe o no sabe, utilizar estrategias de aprendizaje que varían con la naturaleza del material a aprender y las demandas de la situación de aprendizaje, poder predecir y monitorear el éxito de los propios esfuerzos de aprendizaje, entre otras (Roger, Cisero y Carlo, 1993). Algunas estrategias que llevan indirectamente a resultados de aprendizaje son el monitoreo para determinar si el proceso de aprendizaje sucede tal como se planeó, el diagnóstico de las causas de las dificultades, el ajuste de los procesos cuando sea necesario, entre otros (Vermunt, 1996).

Algunos autores enumeran estrategias cognitivas y metacognitivas estableciendo diferencias entre ellas. Sin embargo, otros piensan que la frontera se desdibuja y algunas estrategias pueden desempeorar funciones cognitivas o metacognitivas dependiendo de la situación en que se apliquen (Martí, 1995).

\section{¿CÓMO INDAGAMOS SOBRE LAS ESTRA- TEGIAS COGNITIVAS Y METACOGNITIVAS?}

Nos hemos propuesto estudiar las estrategias que ponen en práctica en la comprensión de textos los estudiantes universitarios de diferentes carreras del mismo rango académico (licenciatura en geografía, profesorado en física, profesorado en química, ingeniería química y bioingeniería). Tratamos de examinar, por un lado, cómo procesan la información proporcionada por el texto por 
Cuadro I

Estrategias cognitivas y metacognitas (fase de evaluación) propuestas en la prueba para ser realizadas a partir del texto proporcionado.

\begin{tabular}{|c|c|c|c|}
\hline \multicolumn{4}{|c|}{ Estrategias cognitivas y metacognitivas (fase de evaluación) } \\
\hline Actividades & Algunos antecedentes & Tipo de actividad & Criterios de evaluación \\
\hline $\begin{array}{l}\text { 1. ¿Cómo ha variado la } \\
\text { temperatura global de la } \\
\text { Tierra en los últimos } \\
\text { años? }\end{array}$ & \multirow{3}{*}{$\begin{array}{l}\text { Diversos investigadores postulan el uso } \\
\text { de cuestionario para estudiar el proce- } \\
\text { samiento que hacen los alumnos de la } \\
\text { información del texto (Otero, 1995; } \\
\text { 1997; Otero y Campanario, 1990; Vi- } \\
\text { dal Abarca, Gilabert y Rouet, 1998). } \\
\text { Atendiendo a estos antecedentes, for- } \\
\text { mulamos las preguntas de las activida- } \\
\text { des } 1,2 \text { y } 3 \text { con el objeto de conocer la } \\
\text { información que los estudiantes han } \\
\text { obtenido del texto. }\end{array}$} & $\begin{array}{l}\text { Comprensión: } \\
\text { búsqueda de informa- } \\
\text { ción específica y res- } \\
\text { puesta a una pregunta. }\end{array}$ & $\begin{array}{l}\text { Evaluamos la detección de la contradicción } \\
\text { que provee el texto. Evaluamos como co- } \\
\text { rrectas las respuestas en que expresan que } \\
\text { la temperatura ha aumentado } 0,5 \mathrm{~K} \text { desde } \\
1900 \text {. }\end{array}$ \\
\hline $\begin{array}{l}\text { 2. ¿A qué causas puede } \\
\text { atribuirse este fenóme- } \\
\text { no? }\end{array}$ & & $\begin{array}{l}\text { Comprensión: } \\
\text { búsqueda de informa- } \\
\text { ción,relación entreideas } \\
\text { y respuesta a una pre- } \\
\text { gunta. }\end{array}$ & $\begin{array}{l}\text { Evaluamos la relación entre el crecimiento } \\
\text { de la población y la industrialización con la } \\
\text { variación de temperatura de la Tierra. Es } \\
\text { correcta la respuesta que indique que las } \\
\text { causas son el crecimiento de la población y } \\
\text { el efecto invernadero (o combinación de } \\
\text { productos gaseosos industriales con } \mathrm{CO}_{2} \\
\text { de la atmósfera). }\end{array}$ \\
\hline $\begin{array}{l}\text { 3. ¿Qué consecuencias } \\
\text { tendríauna variación con- } \\
\text { siderable de la tempera- } \\
\text { tura? }\end{array}$ & & $\begin{array}{l}\text { Comprensión: } \\
\text { búsqueda de informa- } \\
\text { ción,relación entreideas } \\
\text { y respuesta a una pre- } \\
\text { gunta. }\end{array}$ & $\begin{array}{l}\text { Para que la respuesta sea correcta, debería } \\
\text { incluir los efectos en la agricultura, natura- } \\
\text { leza y sociedad humana. }\end{array}$ \\
\hline $\begin{array}{l}\text { 4. ¿Has encontrado al- } \\
\text { gún obstáculo en el texto } \\
\text { que dificulte su compren- } \\
\text { sión? } \\
\quad \text { SÍ NO } \\
\text { Si tu respuesta es afir- } \\
\text { mativa, subraya la difi- } \\
\text { cultad en el texto e indi- } \\
\text { ca en qué consiste. }\end{array}$ & $\begin{array}{l}\text { Otero y Kintsch (1992) han realizado } \\
\text { estudios referidos a los problemas de } \\
\text { comprensión y de control de la com- } \\
\text { prensión con textos breves en los cua- } \\
\text { les han introducido contradicciones } \\
\text { explícitas. Nos interesa averiguar si } \\
\text { los alumnos detectan la contradicción } \\
\text { introducida en el texto. Para hacerlo es } \\
\text { necesario verificar la consistencia ló- } \\
\text { gica de las ideas expresadas en el texto } \\
\text { (criterio de coherencia interna según la } \\
\text { clasificación de Baker, 1994) integrando } \\
\text { información que figura en párrafos di- } \\
\text { ferentes (transpárrafo). }\end{array}$ & $\begin{array}{l}\text { Obstáculos parala com- } \\
\text { prensión: detección de } \\
\text { una contradicción en el } \\
\text { texto. }\end{array}$ & $\begin{array}{l}\text { Evaluamos la proporción de alumnos que } \\
\text { detecta la contradicción y en lo que se } \\
\text { refiere a otras dificultades los resultados se } \\
\text { analizan en función de las respuestas. }\end{array}$ \\
\hline $\begin{array}{l}5 . \text { ¿Hay en el texto pala- } \\
\text { bras que no sabes lo que } \\
\text { significan? Enuméralas. }\end{array}$ & $\begin{array}{l}\text { Nos interesa indagar acerca de la com- } \\
\text { prensión lexical, es decir, que desea- } \\
\text { mos verificar si se ha comprendido el } \\
\text { significado de cada palabra (Baker, } \\
\text { 1994; Otero, 1992). }\end{array}$ & $\begin{array}{l}\text { Criterio lexical: reco- } \\
\text { nocimiento de palabras. }\end{array}$ & $\begin{array}{l}\text { Indagamos en este ítem la relación entre las } \\
\text { palabras que los alumnos incluyen como } \\
\text { desconocidas y la carrera que cursan. }\end{array}$ \\
\hline $\begin{array}{l}\text { 6. ¿Has podido relacio- } \\
\text { nar lo que leíste con algo } \\
\text { que supieras de antes? } \\
\text { ¿Con qué? }\end{array}$ & \multirow{2}{*}{$\begin{array}{l}\text { Baker (1994) indica que en la com- } \\
\text { prensión se produce una contrastación } \\
\text { de la nueva información con lo ya sabi- } \\
\text { do por el lector. Fijamos las activida- } \\
\text { des } 6 \text { y } 7 \text { en las que nos proponemos } \\
\text { indagar si relacionan la nueva infor- } \\
\text { mación con lo que ya saben y con qué } \\
\text { información anterior se produce dicho } \\
\text { vínculo. Además nos interesa conocer } \\
\text { el conflicto entre la información nueva } \\
\text { y la previa. }\end{array}$} & $\begin{array}{l}\text { Coherencia externa: re- } \\
\text { lación de las ideas del } \\
\text { texto con los conoci- } \\
\text { mientos previos. }\end{array}$ & \multirow[t]{2}{*}{$\begin{array}{l}\text { El análisis de las respuestas se ajusta a lo } \\
\text { que expresen los alumnos en las pruebas. } \\
\text { Los criterios son fijados en función de las } \\
\text { respuestas que son abiertas. }\end{array}$} \\
\hline $\begin{array}{l}\text { 7. ¿Hay información en } \\
\text { el texto que no está de } \\
\text { acuerdo con lo que sa- } \\
\text { bías de antes? }\end{array}$ & & $\begin{array}{l}\text { Coherencia externa: re- } \\
\text { lación de las ideas del } \\
\text { texto con los conoci- } \\
\text { mientos previos. }\end{array}$ & \\
\hline $\begin{array}{l}\text { 8. ¿Te parece que al tex- } \\
\text { to le falta información? } \\
\text { ¿Cuál sería? }\end{array}$ & $\begin{array}{l}\text { Otero (1997) sostiene que uno de los } \\
\text { requisitos para considerar que un texto } \\
\text { se comprende es la integridad informa- } \\
\text { tiva que se relaciona con la claridad e } \\
\text { integridad de la información. Consiste } \\
\text { en evaluar si el texto es suficientemen- } \\
\text { te completo y claro. }\end{array}$ & $\begin{array}{l}\text { Integridad, claridad y } \\
\text { suficienciainformativa. } \\
\text { Lectura crítica. }\end{array}$ & $\begin{array}{l}\text { Tratamos de averiguar si los alumnos de- } \\
\text { tectan alguna dificultad en el texto en lo } \\
\text { que se refiere a la falta de información. }\end{array}$ \\
\hline
\end{tabular}


Cuadro II

Estrategias metacognitivas (fase de evaluación) sobre las que se indaga en la prueba.

\begin{tabular}{|c|c|c|c|}
\hline \multicolumn{4}{|c|}{ Estrategias metacognitivas (fase de evaluación) } \\
\hline Actividades & Algunos antecedentes & Tipo de actividad & Criterios de evaluación \\
\hline $\begin{array}{l}\text { 9. Si tu respuesta a la pre- } \\
\text { gunta } 5 \text { ha sido afirmativa, } \\
\text { ¿qué hiciste cuando encon- } \\
\text { traste dichas palabras? } \\
\text { (Puedes marcar más de una } \\
\text { opción.) } \\
\text { - Seguí leyendo. } \\
\text { - Traté de deducir lo que } \\
\text { significan en el contexto. } \\
\text { - Lamenté no tener en este } \\
\text { momento un diccionario } \\
\text { para buscarlas. } \\
\text { - Ninguna de las anterio- } \\
\text { res. } \\
\text { ¿Qué hiciste entonces? }\end{array}$ & $\begin{array}{l}\text { Chamot (1987), Flavell (1976) y } \\
\text { Palincsar y Brown (1996) señalan que } \\
\text { la mejor manera de saber qué estrate- } \\
\text { gias usan los aprendientes mientras rea- } \\
\text { lizan sus tareas de aprendizaje es pre- } \\
\text { guntárselo directamente a ellos. }\end{array}$ & $\begin{array}{l}\text { Evaluación de la com- } \\
\text { prensión(Planolexical) }\end{array}$ & $\begin{array}{l}\text { En la actividad } 5 \text { concentramos nuestra } \\
\text { atenciónen las estrategiasque utilizancuando } \\
\text { no comprenden el significado de algún vo- } \\
\text { cablo. } \\
\text { Contabilizamos las respuestas correspon- } \\
\text { dientes a los tres primeros ítems y elabora- } \\
\text { mos una lista con las opciones diferentes } \\
\text { manifestadas por los estudiantes en el 'úl- } \\
\text { timo ítem. }\end{array}$ \\
\hline $\begin{array}{l}\text { 10. Enumera los pasos que } \\
\text { sigues en general para com- } \\
\text { prender un texto. }\end{array}$ & $\begin{array}{l}\text { En este punto, los estudiantes reflexio- } \\
\text { nan sobre ellos mismos como lectores } \\
\text { describiendo las estrategias que aplican } \\
\text { en una situación de lectura. Algunos } \\
\text { autores usan con este mismo fin las } \\
\text { entrevistas, el procedimiento de pensar } \\
\text { en voz alta o la presentación de casos } \\
\text { para que los alumnos comparen con sus } \\
\text { enfoques de la lectura (Palincsar y Brown, } \\
\text { 1997). }\end{array}$ & $\begin{array}{l}\text { Regulación de la com- } \\
\text { prensión } \\
\text { (Estrategias lectoras) }\end{array}$ & $\begin{array}{l}\text { Nos interesa indagar las estrategias meta- } \\
\text { cognitivas que los alumnos consideran que } \\
\text { ponen en juego en el proceso de compren- } \\
\text { sión. }\end{array}$ \\
\hline
\end{tabular}

medio de un cuestionario de conocimiento y, por otra parte, les planteamos preguntas que apuntan a conocer los obstáculos que han encontrado los estudiantes en la comprensión del texto y las estrategias metacognitivas puestas en juego.

El texto que proporcionamos a los estudiantes fue extraído del ensayo de Krenz «Balance de energía y calentamiento global de la Tierra» que figura al final del capítulo 16 del libro de Física», de Tipler (1994, p. 553). Hemos introducido una contradicción en una de las oraciones a fin de evaluar si los alumnos hacen una lectura crítica. El texto figura en el anexo.

La muestra de estudiantes seleccionada está constituida por 59 alumnos universitarios de los primeros años distribuidos según el siguiente detalle: 31 de la licenciatura en geografía (Grupo 1), 17 de bioingeniería e ingeniería química (Grupo 2) y 11 del profesorado universitario en física y profesorado universitario en química (Grupo 3). La muestra escogida se distribuye en relación con el total de alumnos inscriptos en las carreras seleccionadas. La aplicación de la prueba se llevó a cabo en clases de comprensión de textos científicos.
Cuadro III

Obstáculos que dificultan la comprensión del texto.

\begin{tabular}{|l|c|}
\hline $\begin{array}{l}\text { Dificultades detectadas } \\
\text { por los alumnos }\end{array}$ & $\begin{array}{c}\text { Porcentaje } \\
\text { de alumnos }\end{array}$ \\
\hline Detecta la contradicción & $70 \%$ \\
\hline No sabe el significado de K (grados Kelvin) & $18 \%$ \\
\hline $\begin{array}{l}\text { Indica que el ozono no pertenece a los gases } \\
\text { de la industria }\end{array}$ & $4 \%$ \\
\hline Tiene dificultad con algunas oraciones & $15 \%$ \\
\hline Desconoce el significado de algunas palabras & $11 \%$ \\
\hline
\end{tabular}

Los porcentajes están expresados en función del total de alumnos que encontraron dificultad en la comprensión del texto.

El cuestionario de la prueba, algunos antecedentes, el tipo de actividad y los criterios de evaluación figuran en los cuadros I y II discriminados en estrategias cognitivas y metacognitivas. 
Gráfico 1

Calificación de las respuestas a las preguntas 1, 2 y 3 .

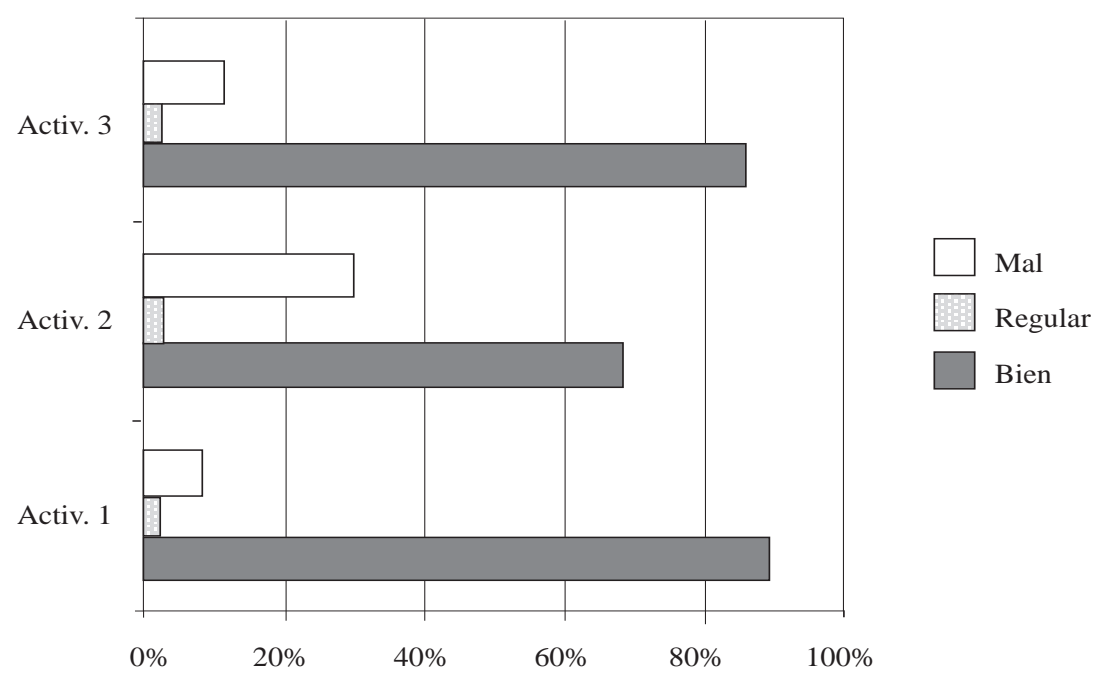

Los resultados están expresados en porcentajes del total de la muestra.

Cuadro IV

Palabras desconocidas por los estudiantes.

\begin{tabular}{|c|c|c|c|c|}
\hline Palabras desconocidas & $\begin{array}{c}\text { Alumnos } \\
\text { de geografía } \\
(\mathbf{N}=31) \\
\%\end{array}$ & $\begin{array}{c}\begin{array}{c}\text { Alumnos de } \\
\text { ingeniería }\end{array} \\
\text { química y bioingeniería } \\
(\mathbf{N}=17) \\
\%\end{array}$ & $\begin{array}{c}\text { Alumnos de física } \\
\text { y química } \\
(\mathbf{N}=11) \\
\%\end{array}$ & $\begin{array}{c}\text { Total de la muestra } \\
\qquad \begin{array}{c}(\mathbf{N}=59) \\
\%\end{array}\end{array}$ \\
\hline Clorofluorcarbonos & 6 & - & 9 & 5 \\
\hline Grado Kelvin (K) & 13 & - & - & 10 \\
\hline Consenso & 13 & 53 & 9 & 24 \\
\hline Permafrost & 16 & 82 & 91 & 49 \\
\hline Concluyentemente & 3 & - & - & 2 \\
\hline Glaciares terrestres & - & 6 & - & 2 \\
\hline
\end{tabular}

Los porcentajes están expresados en función del total de alumnos indicado en cada columna.

\section{RESULTADOS Y SU ANÁLISIS}

Estrategias cognitivas y metacognitivas (fase de evaluación)

\section{Comprensión}

Analizaremos ahora los resultados obtenidos al responder el cuestionario referido a la información del texto.
En lo que se refiere a las actividades 1, 2 y 3, calificamos cada respuesta como buena, regular o mala. Los criterios para el cuestionario están explicados en el cuadro I para respuestas buenas (correctas). Evaluamos como regulares las respuestas que incluyen información parcial y malas cuando son erróneas o están redactadas en forma incoherente. En las preguntas 1 y 3, la información que se requiere para responderlas puede extraerse de un solo párrafo del texto mientras que, para la pregunta 2 , es 
Cuadro V

Vinculación con los conocimientos previos de los estudiantes.

\begin{tabular}{|c|c|c|}
\hline \multicolumn{2}{|c|}{ Conocimientos previos con los que se vinculó la nueva información } & Porcentaje de alumnos \\
\hline \multicolumn{2}{|c|}{ Estudios realizados en otras materias (carrera universitaria) } & 47 \\
\hline \multicolumn{2}{|c|}{ Estudios realizados previamente (secundario) } & 2 \\
\hline \multicolumn{2}{|c|}{ Lecturas previas (diario, libros, artículos varios, revistas, etc.) } & 20 \\
\hline \multicolumn{2}{|c|}{ Medios audiovisuales (TV, charlas, etc.) } & 8 \\
\hline \multirow{9}{*}{ Temas específicos } & Evolución de la Tierra & 2 \\
\hline & Efecto invernadero & 15 \\
\hline & Lluvia ácida & 2 \\
\hline & Ecología & 3 \\
\hline & Ecosistema & 2 \\
\hline & Contaminación ambiental & 3 \\
\hline & Agujero de ozono & 10 \\
\hline & Variaciones climáticas & 2 \\
\hline & Retroceso de glaciares & 2 \\
\hline \multicolumn{2}{|c|}{ No puede establecer relación con conocimientos previos } & 3 \\
\hline \multicolumn{2}{|c|}{ No contesta } & 10 \\
\hline \multicolumn{2}{|c|}{ Respuesta incoherente } & 3 \\
\hline
\end{tabular}

Los porcentajes están expresados en función del total de alumnos de la muestra

necesario relacionar información incluida en diferentes párrafos del texto.

Hemos considerado la muestra total de estudiantes, ya que los porcentajes obtenidos son similares en los tres grupos. Por ejemplo, para las respuestas buenas a la pregunta 1, los resultados para cada grupo son: grupo 1: 90\%; grupo 2: $94 \%$ y grupo 3: $82 \%$. En el gráfico 1 se muestran las calificaciones de las respuestas de los alumnos para cada una de las actividades.

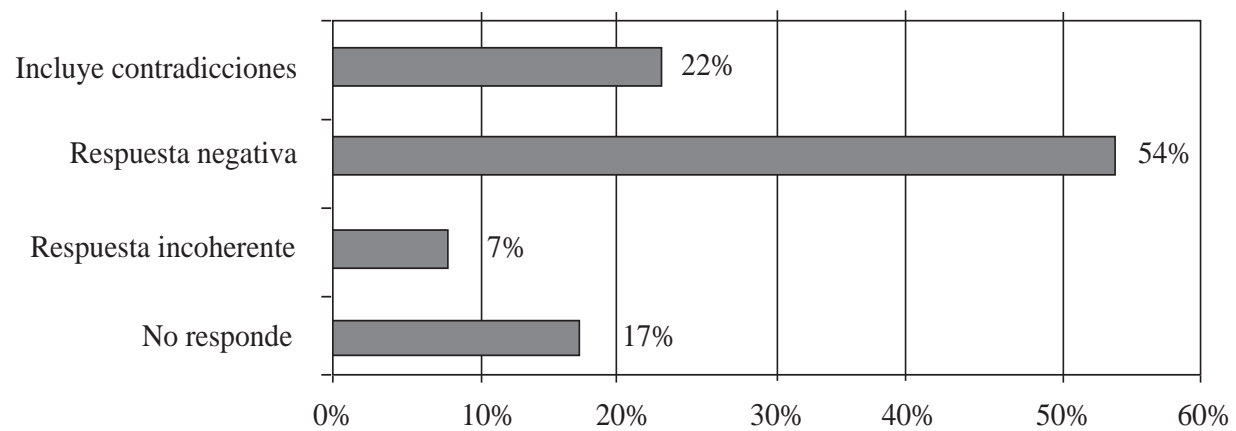

Los resultados están expresados en porcentajes del total de la muestra. 
Observamos que en general esta actividad no ha presentado mayor dificultad, ya que la mayoría de las respuestas es buena y el porcentaje de respuestas regulares es poco significativo. Sin embargo, puede detectarse una diferencia entre las preguntas 1 y 3 con respecto a la 2 .

Hemos calculado la correlación entre los resultados obtenidos para las preguntas 1 y 2 del cuestionario, obteniendo un coeficiente de correlación de Pearson muy bajo $(r=0,23)$. Cuando los alumnos debieron relacionar información transpárrafo (para responder la pregunta 2), algunos no fueron capaces de hacerlo. Es evidente que los estudiantes tienen más facilidad para extraer información en forma literal que para relacionar diferentes ideas.

\section{Obstáculos para la comprensión}

En la prueba, en ningún momento indicamos la existencia de información contradictoria en el texto. La pregunta 4 nos permite saber si se presentaron otras dificultades, además de la contradicción.

Los resultados nos muestran que el $46 \%$ de los estudiantes ha encontrado algún obstáculo que dificulta la comprensión del texto. Los inconvenientes detectados por los alumnos que responden afirmativamente están expresados en el cuadro III. En esta actividad, los alumnos podían mencionar más de una dificultad, por lo que la suma de los porcentajes supera el $100 \%$.

Estos resultados implican que solamente el $32 \%$ de los alumnos encuestados detecta la contradicción introducida en el texto. (Este porcentaje corresponde al $70 \%$ del $46 \%$ del total de la muestra que respondió afirmativamente la pregunta 4.) A pesar de haber fijado la atención en la variación de la temperatura de la Tierra (pregunta 1), no relacionaron en forma exhaustiva las ideas del texto referidas a este tema. Esto evidencia que el obstáculo que presenta el texto no ha sido detectado por un número destacado de estudiantes.

\section{Criterio lexical}

En la actividad 5, los alumnos enumeran las palabras desconocidas por ellos. Las mismas y los porcentajes correspondientes figuran en el cuadro IV.

Consideramos que el número de palabras cuyo significado los alumnos desconocen está en relación inversa con el dominio de vocabulario y que las palabras desconocidas se relacionan con los conocimientos previos. Tal es así que palabras como Kelvin no presentan dificultad para la totalidad de los alumnos de física y química y de ingeniería que estudian física en su formación y sí para los de geografía. La situación opuesta la representan términos como permafrost en la que los porcentajes de desconocimiento por parte de los alumnos son muy altos en ingeniería y los profesorados en física y química, pero notablemente más bajos en geografía. Detectamos además una falta de dominio lexical puesta de manifiesto en el porcentaje destacado de alumnos que desconoce el significado de la palabra consenso.

\section{Coherencia externa}

En la actividad 6 pedimos relacionar lo leído con los conocimientos previos y en la actividad 7, detectar posibles diferencias entre la nueva información y los conocimientos previos.

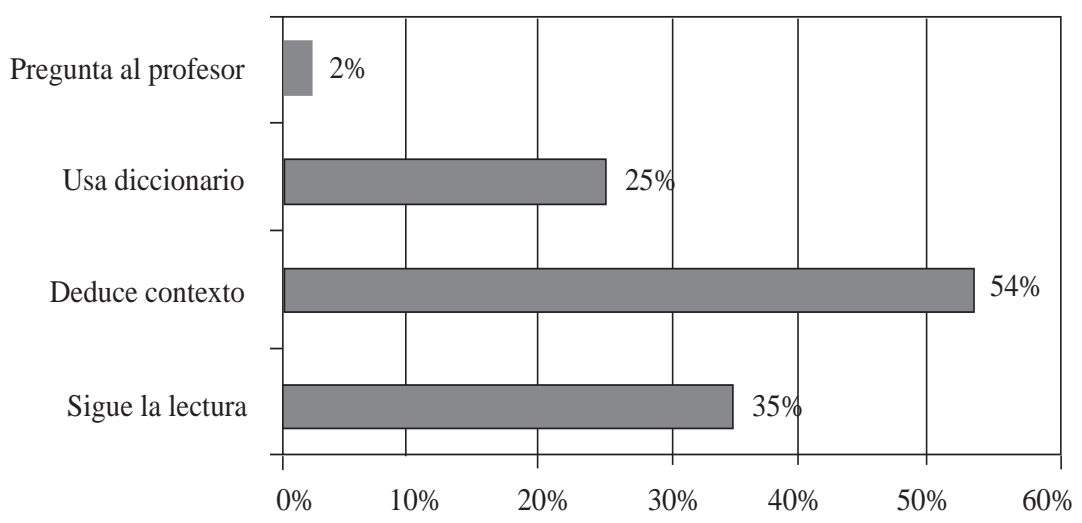

Los resultados están expresados en porcentajes del total de la muestra. 
Sobre la base del análisis realizado a partir de las respuestas de los alumnos, hemos agrupado las vinculaciones con los conocimientos previos tal como se muestra en el cuadro $\mathrm{V}$.

Con respecto a la actividad 7 , en que pedimos identificar aquella información del texto que se contradice con los conocimientos previos, las respuestas de los estudiantes las clasificamos en: incluye la contradicción, no encuentra información contradictoria (respuesta negativa), no responde o lo hace en forma incoherente. En el gráfico 2 aparecen los resultados obtenidos y se indican los porcentajes para cada una de las respuestas.

\section{Integridad, claridad y suficiencia informativa}

En la actividad 8, los alumnos indican si han detectado alguna dificultad en el texto. La pregunta es: « $i$ Te parece que al texto le falta información? ¿Cuál sería?» Encontramos que el $30 \%$ de los estudiantes indica que no le falta información al texto, el $15 \%$ no contesta, el $8 \%$ lo hace en forma incoherente y el $47 \%$ restante señala algunas falencias en el texto. Entre éstas se destacan, por haberse reiterado al menos una vez, que el mismo omite comentarios sobre la concienciación de la población para solucionar el problema, las consecuencias para la salud y para el medio ambiente y que le faltaría profundidad. Sólo un alumno incluye la contradicción en su respuesta a esta pregunta. Este alumno da por cierta la afirmación de que la temperatura aumenta (en todas las respuestas anteriores acepta este hecho), pero al responder esta actividad indica que le falta información para justificar después por qué disminuye la temperatura. En este caso, para salvar la contradicción, el lector hace una inferencia que adjudica falta de información al texto.

\section{Estrategias metacognitivas (fase de regulación)}

\section{Regulación de la comprensión (plano lexical)}

En la actividad 9 nos referimos a las estrategias usadas cuando los estudiantes encontraron en el texto palabras cuyo significado desconocen. Las opciones son: seguir la lectura, deducir del contexto, lamentar no tener diccionario para consultarlo y otras entre las cuales los alumnos incluyeron la pregunta al profesor. Hemos indicado los resultados obtenidos en el gráfico 3 .

Los resultados nos muestran que los estudiantes, en su mayoría, no tratan de buscar solución preguntando al profesor o buscando en el diccionario, sino que reparan la situación encontrando respuestas dentro del mismo texto, lo que ayudaría a tender a un aprendizaje autónomo.

\section{Regulación de la comprensión (estrategias lectoras)}

En la actividad 10, los alumnos deben mencionar cuáles son los pasos que siguen, por lo general, cuando leen un texto. Las categorías de los pasos seguidos para comprender un texto han surgido de la lectura de las respues- tas de los alumnos y podemos agruparlas según los siguientes criterios:

- Primer acercamiento al material de lectura. En este punto, los alumnos manifiestan diferentes respuestas:

a) La mayoría lee primero el texto en forma completa y posteriormente relee varias veces.

b) Otro grupo manifiesta que hace una lectura deteniéndose a analizar párrafo por párrafo (lo cual en muchos casos aparece como paso siguiente a la lectura completa del texto).

c) Una minoría da primero un vistazo general al texto en forma rápida como paso previo a la lectura.

- Solución de dificultades en el plano lexical. El 40\% de los estudiantes indica en sus respuestas que trata de solucionar las dificultades en el plano lexical (ya sea buscando en el diccionario o consultando al profesor o a sus compañeros). Esta actividad se incluye en la mayoría de los casos como segundo o tercer paso en orden de ejecución.

- Jerarquización de ideas. Incluimos en esta categoría la identificación de las ideas principales y secundarias, ya sea subrayando en el texto o extrayendo las ideas importantes o las ideas importantes y las secundarias. El 56\% de los estudiantes indicaron en sus respuestas que usan estrategias de este tipo.

- Otras estrategias mencionadas con menor frecuencia se refieren a: elaborar un resumen o revisar, realizar un esquema, discutir con otros, hacerse preguntas sobre el texto o comentar las ideas en voz alta, entre otras.

\section{A MODO DE CONCLUSIÓN}

El análisis de los resultados de la prueba nos ha permitido recabar información sobre la forma en que los estudiantes universitarios utilizan algunas estrategias cognitivas y metacognitivas. Los resultados, en general, nos muestran que la comprensión del texto es limitada y, por lo tanto, no está acorde con lo que esperaríamos para alumnos de este nivel en lo que se refiere a la manera de comprender y extraer información de un texto expositivo.

Es importante destacar que no hay una correlación fuerte entre algunas de las actividades propuestas. Esto pone de manifiesto el escaso número de buenos lectores, capaces de realizar todas las actividades en forma correcta. Lo mismo ocurre para los que presentan deficiencias estratégicas de lectura y comprensión.

El porcentaje de alumnos que manifiesta haber encontrado contradicción en el texto es inferior a los que indican haber tenido obstáculos en su comprensión. El hecho de que los estudiantes no detecten en su mayoría la contradicción parece sugerir que las relaciones semánticas que 
son necesarias para la coherencia de un fragmento del texto no son necesariamente computadas por el lector (Van Ostendorp y Zwaan, 1994). Algunas de las causas que podemos aducir es que la contradicción está en un párrafo distante (transpárrafo) de la información correcta o que hay falta de dominio de integración de lo que se lee, es decir, de la consideración global de la información del texto. También podría deberse a la autoridad que se le asigna a la palabra escrita, proporcionada por el docente, que desanima al alumno a discrepar de ella.

La dificultad léxica puede obstaculizar el aprendizaje de los estudiantes, ya que rompe la coherencia del texto para el lector con dificultades en el ámbito lexical (León y Slisko, 2000). Ha surgido en el análisis que algunos alumnos desconocen el significado de palabras usuales del lenguaje, lo que muestra pobreza de vocabulario (por ejemplo, consenso y concluyentemente). La cantidad de palabras enumeradas como desconocidas nos lleva a pensar en una comprensión «fraccionada» y producida en un plano meramente lexical sin posibilidad de una comprensión global.

Como consecuencia de este análisis reflexivo de los resultados, creemos importante que los docentes sepamos guiar a nuestros estudiantes en la adquisición e implementación de actividades que les permitan mejor dominio de los textos tanto en los aspectos cognitivos como metacognitivos. Flavell (1996) afirma que «cuando pensamos en el desarrollo cognitivo pensamos naturalmente en la adquisición de nuevas destrezas y conocimientos. Sin embargo, el desarrollo cognitivo consiste también en el incremento posterior de los conocimientos y destrezas que ya existen en el repertorio (p. 166).» Teniendo en cuenta esto, la investigación nos permite afirmar que las dificultades de nuestros estudiantes son serias y que debemos intervenir en consecuencia.

Proponer actividades cognitivas y metacognitivas a partir de la lectura de textos científicos nos permitirá contribuir al desarrollo de ciertas competencias lectoras de nuestros alumnos. Al respecto, Flavell (1996) indica que «a medida que se desarrolla una competencia puede convertirse en algo a lo que se accede y se utiliza en forma más fiable en cualquier tarea dada que lo exija» (p. 167). Es decir, que, en la medida que propongamos a nuestros estudiantes la aplicación de estrategias cognitivas y metacognitivas, mejor será la utilización que ellos hagan de las mismas.

Roger, Cisero y Carlo (1993) indican que el que tiene habilidades en un área de conocimiento tiene habilidades metacognitivas para operar en esa área. Sobre esta base, nuestra intervención no debe limitarse únicamente a las estrategias cognitivas sino que debería incluir, además actividades de evaluación y regulación de la comprensión. Es así que debemos pensar en seleccionar aquellas actividades que ayuden a los alumnos universitarios a mejorar su comprensión a partir de textos y además a analizar en qué medida las estrategias que han aplicado pueden ser mejoradas y les permitan procesar adecuadamente la información.

Creemos que es importante, para la comprensión y aprendizaje que logren los alumnos a partir de los textos, que realicen un buen uso de estrategias metacognitivas. La utilidad de la aplicación de las mismas está en función de que el alumno logre evaluar y regular su propia comprensión. Consideramos que los mecanismos que se ponen en juego en estos procesos hacen necesario que los docentes nos comprometamos activamente instruyendo a los estudiantes en la adquisición e implementación de estrategias que apunten a un dominio en los aspectos cognitivos y metacognitivos, ambos estrechamente vinculados.

\section{AGRADECIMIENTO}

Damos las gracias a la Universidad Nacional de San Juan (República Argentina), que avala y subsidia las investigaciones realizadas en el Proyecto núm. 21/F166.

\section{REFERENCIAS BIBLIOGRÁFICAS}

BAKER, L. (1994). Metacognición, lectura y educación científica, en Minnick Santa, C. y Alvermann, D.E. (comp.). Una didáctica de las ciencias, procesos y aplicaciones. Argentina: Aique.

BLYTHE, T. y PERKINS, D. (1999). Comprender la comprensión, en Blythe, T. (comp.). La enseñanza para la comprensión. Guía para el docente. Buenos Aires: Paidós.

BROWN A.L., ARMBRUSTER, B.B. y BAKER, L. (1986). The Role of Metacognition in Reading and Studying, Reading Comprehension: From Research to Practice. Ora, J. (ed.). Nueva York. LEA: Hillsdale.

CAMPANARIO, J.M. (1994). Los problemas crecen: a veces los alumnos no se enteran de que no se enteran. X Encuentro sobre Aspectos Didácticos en laEnseñanza Secundaria. España. 
CHAMOT, A.H. (1987). The learning strategies of the students of English as a Second Language, en Wenden, A. y Rubin, J., Learner Strategies in Language Learning. Teaching Methodology Series, Prentice Hall International.

DE VEGA, M. (1993). Introducción a la psicología cognitiva. Madrid: Alianza.

DICCIONARIO DE LA REAL ACADEMIA. (1992). Editorial Espasa Calpe, SA.

FLAVELL, J.H. (1976). Metacognitive Aspects of Problem Solving, en Resnick L.B. (ed.). The Nature of Intelligence. Hillsdale. Nueva York: Lawrence Erlbaum.

FLAVELL, J. (1996). El desarrollo cognitivo. España: Prentice Hall.

GELLATLY, A. (1997). La inteligencia hábil. El desarrollo de las capacidades cognitivas. Buenos Aires: Aique.

LEÓN, J.A. y SLISKO, J. (2000). La dificultad comprensiva de los textos de ciencias. Nuevas alternativas para un viejo problema educativo. Psicología Educativa, 6 (1), pp. 7-26.

LUQUE, J. L., GARCÍA MADRUGA, J.A., GUTIÉRREZ, F., ELOSÚA M.R. y GÁRATE, M. (1999). La construcción de la representación semántica de los textos, en Comprensión lectora y memoria operativa. Aspectos evolutivos e instruccionales. Barcelona: Paidós.

MARTÍ, E. (1995). Metacognición: entre la fascinación y el desencanto. Infancia y Aprendizaje, 72, pp. 9-32.

OTERO, J. (1990). Variables cognitivas y metacognitivas en la comprensión de textos científicos: el papel de los esquemas y el control de la propia comprensión, Enseñanza de las Ciencias, 8(1), pp. 17-22.

OTERO, J. (1992). El aprendizaje receptivo de las ciencias: preconcepciones, estrategias cognitivas y estrategias metacognitivas. Tarbiya, 2, pp. 57-65.

OTERO, J. (1995). Estrategias básicas de aprendizaje frente a contenidos y métodos en la enseñanza de la física. Tarbiya, 10, pp. 127-133.

OTERO, J. (1997). El conocimiento de la falta de conocimiento de un texto científico. Alambique, 11, pp. 15-22.

OTERO, J. y CAMPANARIO, J.M. (1990). Comprehension evaluation and regulation in learning from science texts. Journal of Research in Science Teaching, 27(5), pp. 447460.

OTERO, J. y KINTSCH, W. (1992). Failures to Detect Contradictions in a Text: What readers believe versus what they read. Psychological Science, 3(4), pp. 229-235.

PALINCSAR, A.S. y BROWN, A.L. (1997). La enseñanza para la lectura autorregulada, en Resnick L. y Klopfer L. (comp.) Currículum y cognición. Argentina: Aique.

ROYER, J.M., CISERO, C.A. y CARLO, M.S. (1993). Techniques and Procedures for Assessing Cognitive Skills, Review of Educational Research, 63(2), pp. 201-243.

TIPLER, P. (1994). Física. España: Reverté, SA.

VAN OSTENDORP, H. y ZWAAN, R. A. (1994). Naturalistic Text Comprehension. Nueva Jersey: Ablex Publishing Corporation.

VERMUNT, J.D. (1996). Metacognitive, cognitive and affective aspects of learning strategies: A phenomenographic analysis. Higher Education, 31, pp. 25-50. Kluwer Academic Publishers.

VIDAL, E., GILABERT, R. y ROUET, J.F. (1998). El papel de las preguntas en el aprendizaje a partir de textos científicos. Documento del trabajo presentado en el Seminario de Comprensión y Producción de Textos Científicos. Aveiro. Portugal.

[Artículo recibido en noviembre de 2000 y aceptado en abril de 2002.] 


\section{ANEXO}

Los seres humanos han habitado este planeta durante millones de años, pero sólo durante los últimos cien años aproximadamente - como consecuencia tanto del crecimiento de la población como de su industrialización- empezaron a influir sobre nuestro clima. La temperatura global ha aumentado en 0,5 K desde 1900, los niveles de los océanos han subido, los glaciares terrestres están retrocediendo, las capas de hielo polares se están reduciendo y la profundidad del permafrost en Alaska ha disminuido. La combustión de los combustibles fósiles y la deforestación se combinan para producir un incremento de los niveles atmosféricos de dióxido de carbono $\left(\mathrm{CO}_{2}\right.$ ). Este gas, combinado con otros productos gaseosos de la industria (por ejemplo, el óxido nitroso, los clorofluorcarbonos y el ozono), ha promovido un calentamiento global de la Tierra, debido a un proceso comúnmente denominado efecto invernadero.

Todavía se está debatiendo si los modelos climatológicos utilizados en la actualidad prueban concluyentemente que se está produciendo un calentamiento global creciente. Sin embargo, existe un consenso en la comunidad científica de que una continuación de las actividades humanas actuales dará como resultado una disminución de la temperatura (de 1,5 K a 5,5 K) en los próximos cincuenta a cien años. Un calentamiento global de esta magnitud tendría efectos profundos y de larga duración sobre la agricultura, la propia naturaleza y la sociedad humana. 